\title{
Pragmatic Improvement of Magnetic Exchange Couplings from Subsystem Density-Functional Theory through Orthogonalization of Subsystem Orbitals - Supporting Information -
}

\author{
Anja Massolle and Johannes Neugebauer ${ }^{[}$ \\ Theoretische Organische Chemie, Organisch-Chemisches Institut \\ and Center for Multiscale Theory and Computation, \\ Westfälische Wilhelms-Universität Münster \\ Corrensstraße 36, 48149 Münster, Germany
}

Date: $\quad$ February 16, 2021

${ }^{1}$ email: j.neugebauer@uni-muenster.de 


\section{S1 Lewis Structures}

In Fig. S1 the Lewis formulas of the radical monomers employed in this work are shown.

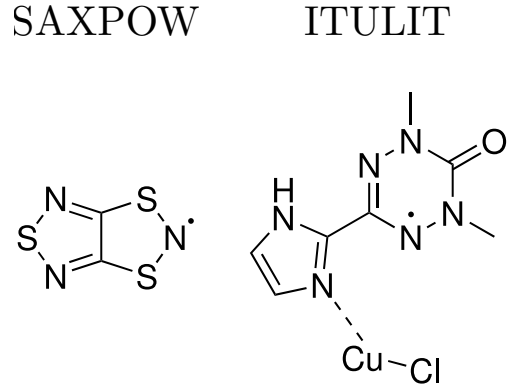

1-3

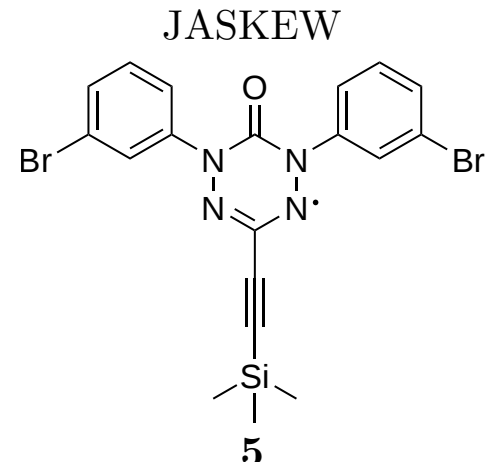

DESJET<smiles>N#CC(C#N)c1cc2c3c(c1)CCCN3CCC2</smiles>

6

Figure S1: Lewis structures of the radical monomers studied in this work. The corresponding CCDC identifier is listed above the Lewis structure. 


\section{S2 Density Error Introduced by the Orthogonalization}

The integrated spin density error introduced by the Löwdin orthogonalization procedure is shown in Fig S2 to S6 for compounds 2-6.

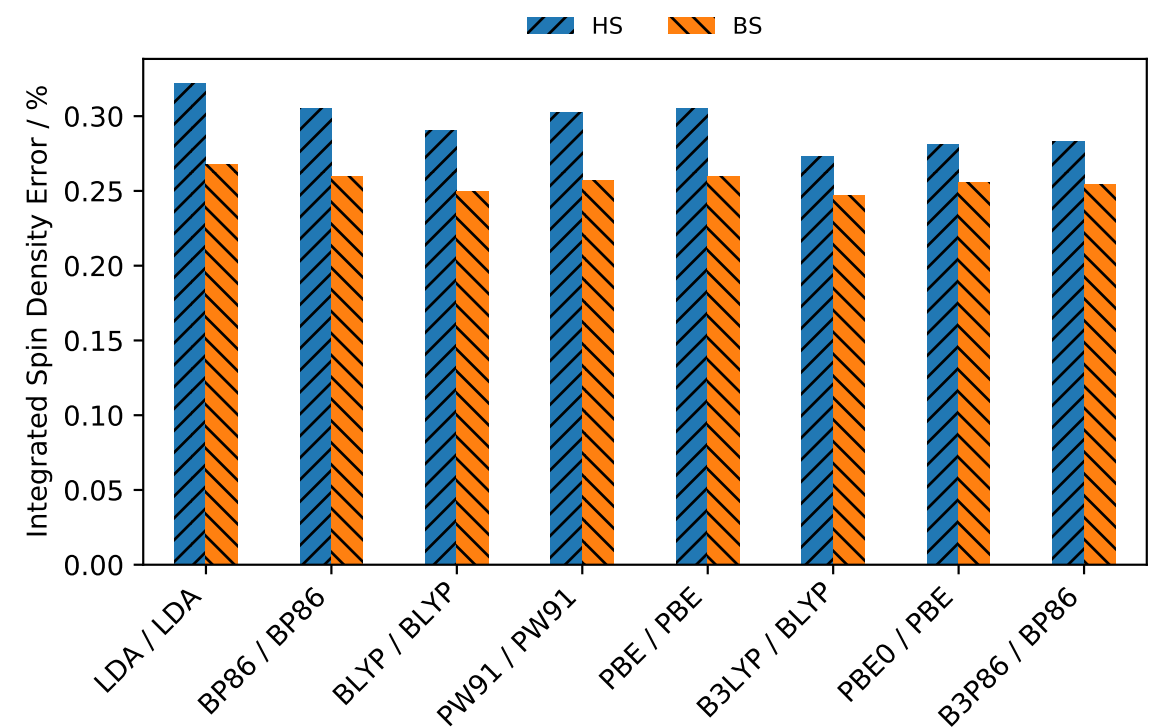

Figure S2: Integrated spin density error for compound $\mathbf{2}$ after applying the Löwdin orthogonalization. The XC / nad XC functional combination employed in the sDFT calculation is shown on the $x$ axis. 


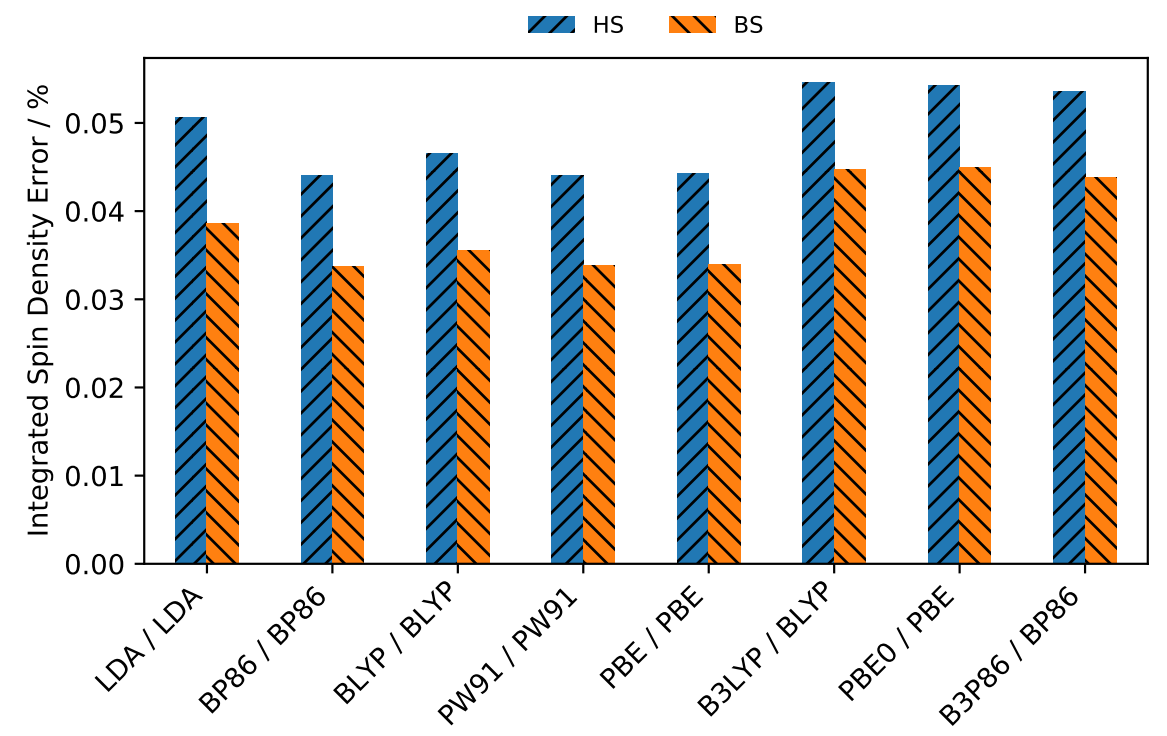

Figure S3: Integrated spin density error for compound 3 after applying the Löwdin orthogonalization. The XC / nad XC functional combination employed in the sDFT calculation is shown on the $x$ axis.

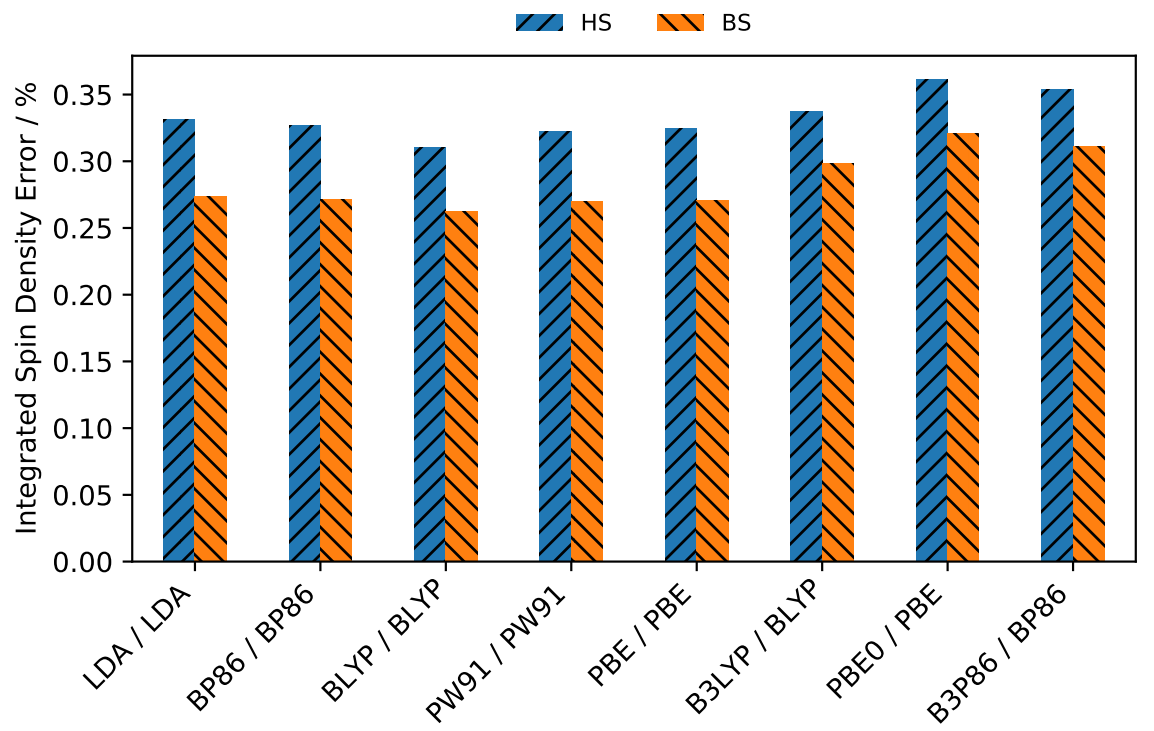

Figure S4: Integrated spin density error for compound 4 after applying the Löwdin orthogonalization. The XC / nad XC functional combination employed in the SDFT calculation is shown on the $x$ axis. 


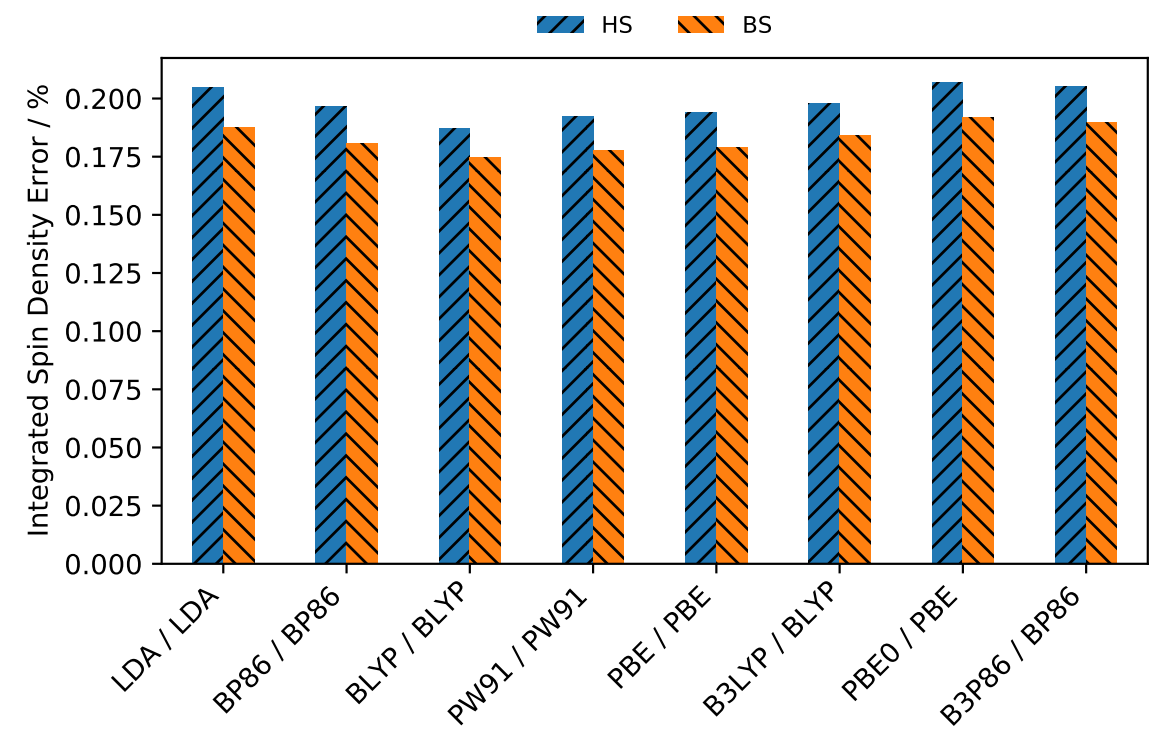

Figure S5: Integrated spin density error for compound $\mathbf{5}$ after applying the Löwdin orthogonalization. The XC / nad XC functional combination employed in the sDFT calculation is shown on the $x$ axis.

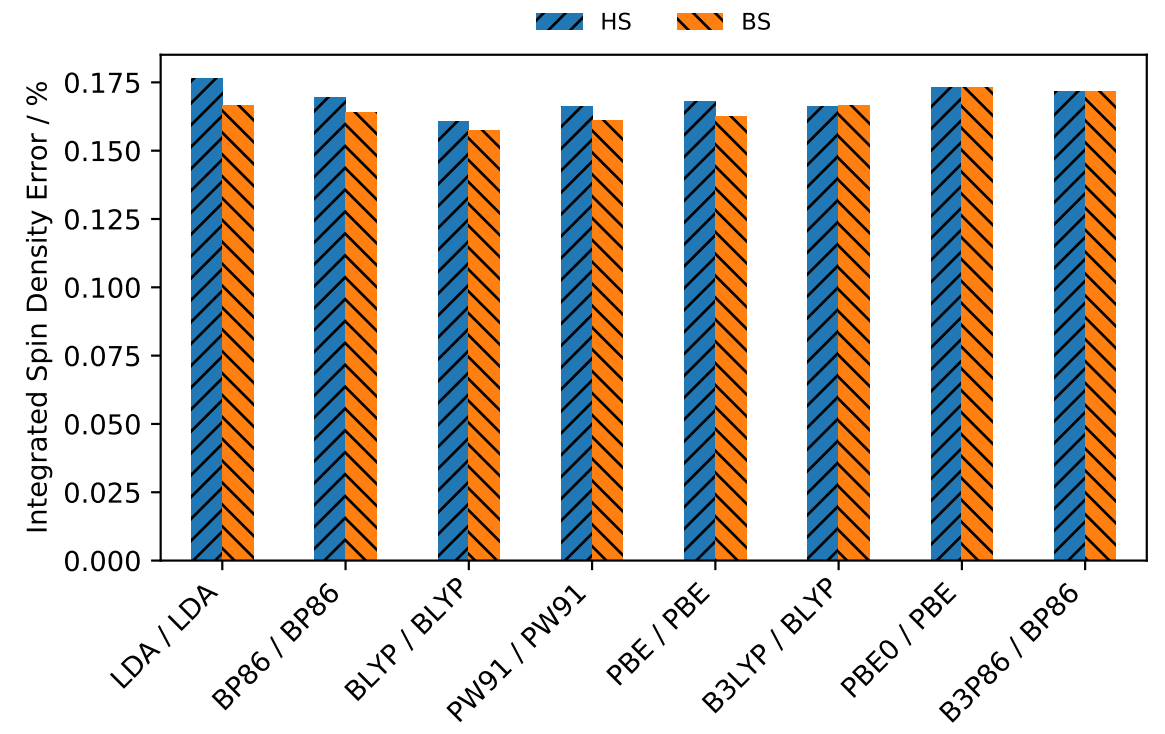

Figure S6: Integrated spin density error for compound 6 after applying the Löwdin orthogonalization. The XC / nad XC functional combination employed in the SDFT calculation is shown on the $x$ axis. 


\section{S3 Other Orthogonalization Techniques}

To test the numeric stability of different orthogonalization techniques we calculated the magnetic coupling constant $J$ with four different procedures.

Löwdin Löwdins symmetric orthogonalization ${ }^{1]}$ as described in the main article.

Pipek Pipeks iterative procedure which tries to keep the orbitals as local as possible. ${ }^{2}$

Broer Broers orthogonalization method which is based on corresponding orbitals..$^{3}$

$\mathbf{T}_{\text {kin }}^{\text {non-ortho }}$ The kinetic energy can also be calculated from non-orthogonal orbitals by expressing the density matrix in the following way: $\underline{4}$

$$
P=C S^{-1} C^{\top}
$$

The kinetic energy of the supersystem can then be evaluated as:

$$
E_{\text {kin }}=\sum_{i, j}^{n_{\text {bas }}} P_{i j}\left\langle\chi_{i}(\mathbf{r})\left|-\frac{\nabla^{2}}{2}\right| \chi_{j}(\mathbf{r})\right\rangle
$$

Where $\chi(\mathbf{r})$ are the AO basis functions and $P$ is the density matrix from Eq. (S1).

In Tab. S1 the magnetic exchange coupling constants calculated with method B (only $T_{s}^{\text {nad }}$ is calculated from the orthogonal orbitals) and the four orthogonalization procedures are listed. 
Table S1: Mean values and standard deviations of $J$ (in units of $\mathrm{cm}^{-1}$ ) calculated with the $\mathrm{XC}$-functionals listed in the computational details for all investigated systems. When sDFT was employed $T_{s}^{\text {nad }}$ was either calculated from a NAKE functional or by orthogonalized subsystem orbitals. Method B was employed for the energy evaluation via orthogonalized orbitals.

\begin{tabular}{llrrrrrr}
\hline & & $\mathbf{1}$ & $\mathbf{2}$ & $\mathbf{3}$ & $\mathbf{4}$ & $\mathbf{5}$ & $\mathbf{6}$ \\
\hline \multirow{2}{*}{ KS-DFT } & mean & -1696 & -195 & -19 & -833 & -499 & -6 \\
& std & 199 & 85 & 9 & 216 & 154 & 7 \\
\hline \multirow{2}{*}{ sDFT, NAKE } & mean & -61 & -10 & 0 & -27 & -11 & -3 \\
& std & 4 & 2 & 0 & 3 & 2 & 1 \\
\hline \multirow{2}{*}{ sDFT, Löwdin } & mean & -2724 & -162 & -10 & -845 & -406 & -12 \\
& std & 46 & 19 & 1 & 168 & 26 & 1 \\
\hline \multirow{2}{*}{ sDFT, Pipek } & mean & -2724 & -162 & -10 & -845 & -406 & -12 \\
& std & 46 & 19 & 1 & 168 & 26 & 1 \\
\hline \multirow{2}{*}{ sDFT, Broer } & mean & -2724 & -162 & -10 & -845 & -406 & -12 \\
& std & 46 & 19 & 1 & 168 & 26 & 1 \\
\hline \multirow{2}{*}{ sDFT, T $T_{\text {kin }}^{\text {non-otho }}$} & mean & -2724 & -162 & -10 & -845 & -406 & -12 \\
& std & 46 & 19 & 1 & 168 & 26 & 1 \\
\hline
\end{tabular}




\section{S4 Magnetic Exchange Coupling Constants}

In Tab. S2 to S6 the magnetic exchange coupling constants calculated for compounds 2-6 with KS-DFT and different sDFT methods are compiled.

Table S2: Magnetic exchange coupling constants (in units of $\mathrm{cm}^{-1}$ ) evaluated with the PW91k NAKE functional, without any NAKE contribution and via method A and B for Löwdin orthogonalized orbitals for compound $\mathbf{2}$.

\begin{tabular}{llrrrrr}
\hline \hline XC & nad XC & NAKE & $T_{s}^{\text {nad }}=0$ & Method A $($ all $)$ & Method B $\left(T_{s}^{\text {nad }}\right)$ & KS-DFT \\
\hline LDA & LDA & -6 & 5 & -18 & -188 & -328 \\
BP86 & BP86 & -12 & -1 & -21 & -174 & -237 \\
BLYP & BLYP & -13 & -3 & -22 & -168 & -225 \\
PW91 & PW91 & -10 & 0 & -20 & -172 & -230 \\
PBE & PBE & -10 & 0 & -21 & -175 & -236 \\
B3LYP & BLYP & -11 & 0 & -19 & -140 & -104 \\
PBE0 & PBE & -9 & 2 & -17 & -138 & -93 \\
B3P86 & BP86 & -10 & 2 & -18 & -143 & -105 \\
\hline \hline
\end{tabular}

Table S3: Magnetic exchange coupling constants (in units of $\mathrm{cm}^{-1}$ ) evaluated with the PW91k NAKE functional, without any NAKE contribution and via method A and B for Löwdin orthogonalized orbitals for compound $\mathbf{3}$.

\begin{tabular}{llrrrrr}
\hline \hline XC & nad XC & NAKE & $T_{s}^{\text {nad }}=0$ & Method A $($ all $)$ & Method B $\left(T_{s}^{\text {nad }}\right)$ & KS-DFT \\
\hline LDA & LDA & 0 & 0 & -2 & -12 & -33 \\
BP86 & BP86 & 0 & 0 & -2 & -11 & -23 \\
BLYP & BLYP & 0 & 0 & -2 & -11 & -23 \\
PW91 & PW91 & 0 & 0 & -2 & -11 & -22 \\
PBE & PBE & 0 & 0 & -2 & -11 & -23 \\
B3LYP & BLYP & 0 & 0 & -1 & -9 & -9 \\
PBE0 & PBE & 0 & 0 & -1 & -8 & -7 \\
B3P86 & BP86 & 0 & 0 & -1 & -9 & -9 \\
\hline \hline
\end{tabular}


Table S4: Magnetic exchange coupling constants (in units of $\mathrm{cm}^{-1}$ ) evaluated with the PW91k NAKE functional, without any NAKE contribution and via method A and B for Löwdin orthogonalized orbitals for compound 4 .

\begin{tabular}{llrrrrr}
\hline \hline XC & nad XC & NAKE & $T_{s}^{\text {nad }}=0$ & Method A (all) & Method B $\left(T_{s}^{\text {nad }}\right)$ & KS-DFT \\
\hline LDA & LDA & -22 & 10 & -131 & -692 & -940 \\
BP86 & BP86 & -26 & 7 & -138 & -746 & -1106 \\
BLYP & BLYP & -30 & 1 & -139 & -729 & -1088 \\
PW91 & PW91 & -25 & 8 & -134 & -732 & -530 \\
PBE & PBE & -26 & 7 & -135 & -723 & -548 \\
B3LYP & BLYP & -31 & 17 & -171 & -1019 & -843 \\
PBE0 & PBE & -27 & 28 & -174 & -1072 & -773 \\
B3P86 & BP86 & -27 & 24 & -170 & -1047 & -839 \\
\hline \hline
\end{tabular}

Table S5: Magnetic exchange coupling constants (in units of $\mathrm{cm}^{-1}$ ) evaluated with the PW91k NAKE functional, without any NAKE contribution and via method A and B for Löwdin orthogonalized orbitals for compound $\mathbf{5}$.

\begin{tabular}{llrrrrr}
\hline \hline XC & nad XC & NAKE & $T_{s}^{\text {nad }}=0$ & Method A $($ all $)$ & Method B $\left(T_{s}^{\text {nad }}\right)$ & KS-DFT \\
\hline LDA & LDA & -7 & 5 & -56 & -387 & -731 \\
BP86 & BP86 & -11 & 1 & -59 & -394 & -584 \\
BLYP & BLYP & -14 & -2 & -60 & -382 & -569 \\
PW91 & PW91 & -11 & 2 & -58 & -388 & -562 \\
PBE & PBE & -11 & 2 & -57 & -390 & -571 \\
B3LYP & BLYP & -13 & 5 & -67 & -426 & -337 \\
PBE0 & PBE & -10 & 10 & -68 & -444 & -305 \\
B3P86 & BP86 & -11 & 8 & -66 & -438 & -336 \\
\hline \hline
\end{tabular}

Table S6: Magnetic exchange coupling constants (in units of $\mathrm{cm}^{-1}$ ) evaluated with the PW91k NAKE functional, without any NAKE contribution and via method A and B for Löwdin orthogonalized orbitals for compound 6 .

\begin{tabular}{llrrrrr}
\hline \hline XC & nad XC & NAKE & $T_{s}^{\text {nad }}=0$ & Method A $($ all $)$ & Method B $\left(T_{s}^{\text {nad }}\right)$ & KS-DFT \\
\hline LDA & LDA & -1 & 2 & 0 & -8 & -14 \\
BP86 & BP86 & -3 & -1 & -3 & -12 & -10 \\
BLYP & BLYP & -4 & -2 & -3 & -12 & -12 \\
PW91 & PW91 & -3 & -1 & -2 & -11 & -10 \\
PBE & PBE & -3 & -1 & -2 & -12 & -9 \\
B3LYP & BLYP & -3 & -1 & -2 & -12 & 1 \\
PBE0 & PBE & -2 & -1 & -1 & -12 & 3 \\
B3P86 & BP86 & -3 & -1 & -2 & -12 & 2 \\
\hline \hline
\end{tabular}


The magnetic exchange coupling constants of $\mathbf{1}$ to $\mathbf{6}$ calculated with sDFT using the PW91k NAKE functional, orthogonalized orbitals and KS-DFT are depicted in Fig. S7 to S12

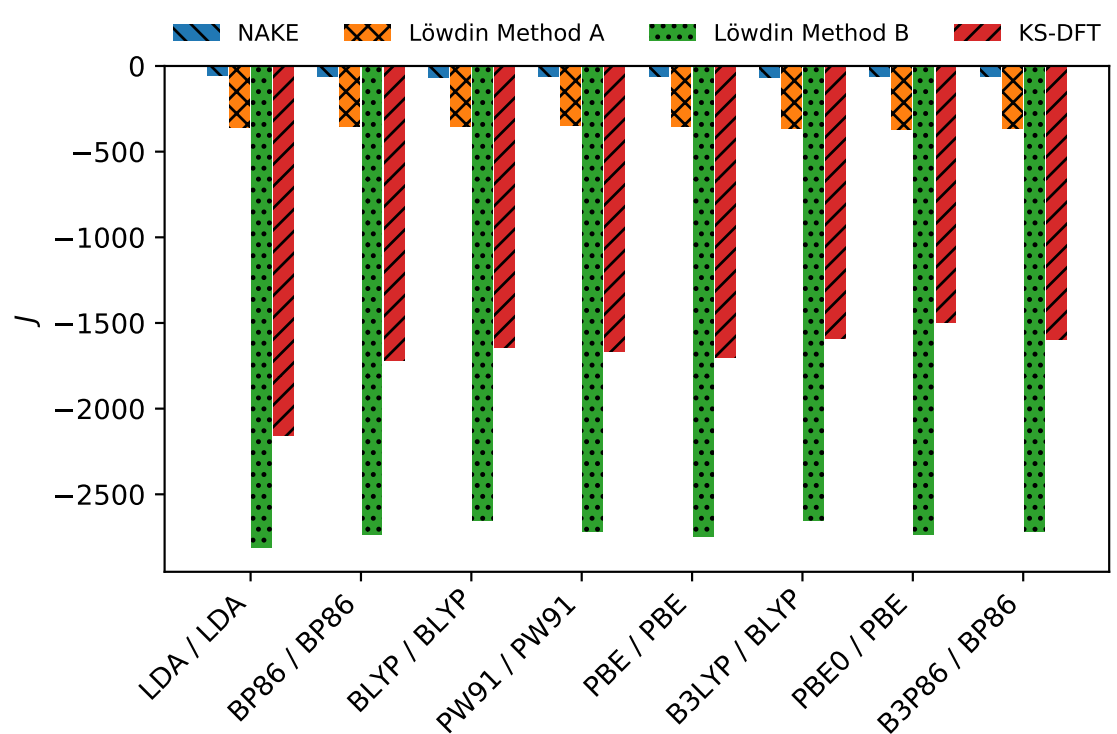

Figure S7: Magnetic exchange coupling constants evaluated for compound 1 with different schemes for the calculation of $T_{s}^{\text {nad }}$ and various XC / nad XC functionals. NAKE: $T_{s}^{\text {nad }}$ evaluated with a NAKE functional and Löwdin: $T_{s}^{\text {nad }}$ evaluated from Löwdin orthogonalized orbitals via method A or B. The corresponding KS-DFT values calculated with the listed XC functional are shown for reference. 


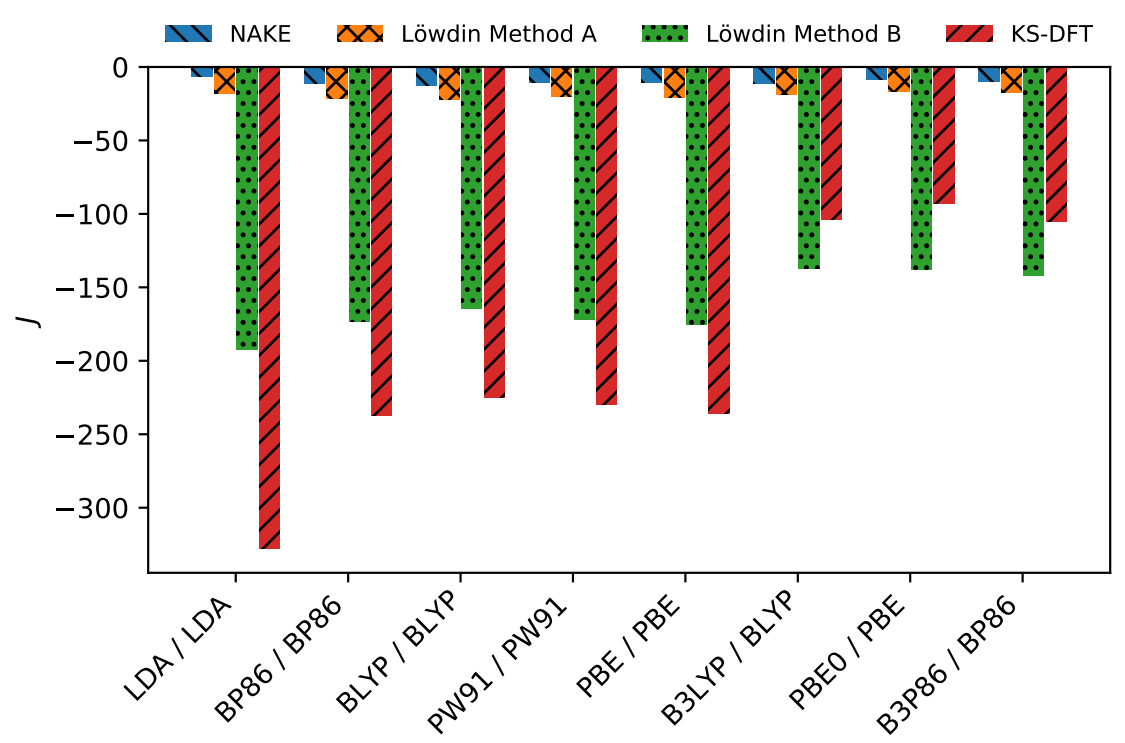

Figure S8: Magnetic exchange coupling constants evaluated for compound $\mathbf{2}$ with different schemes for the calculation of $T_{s}^{\text {nad }}$ and various XC / nad XC functionals. NAKE: $T_{s}^{\text {nad }}$ evaluated with a NAKE functional and Löwdin: $T_{s}^{\text {nad }}$ evaluated from Löwdin orthogonalized orbitals via method A or B. The corresponding KS-DFT values calculated with the listed XC functional are shown for reference.

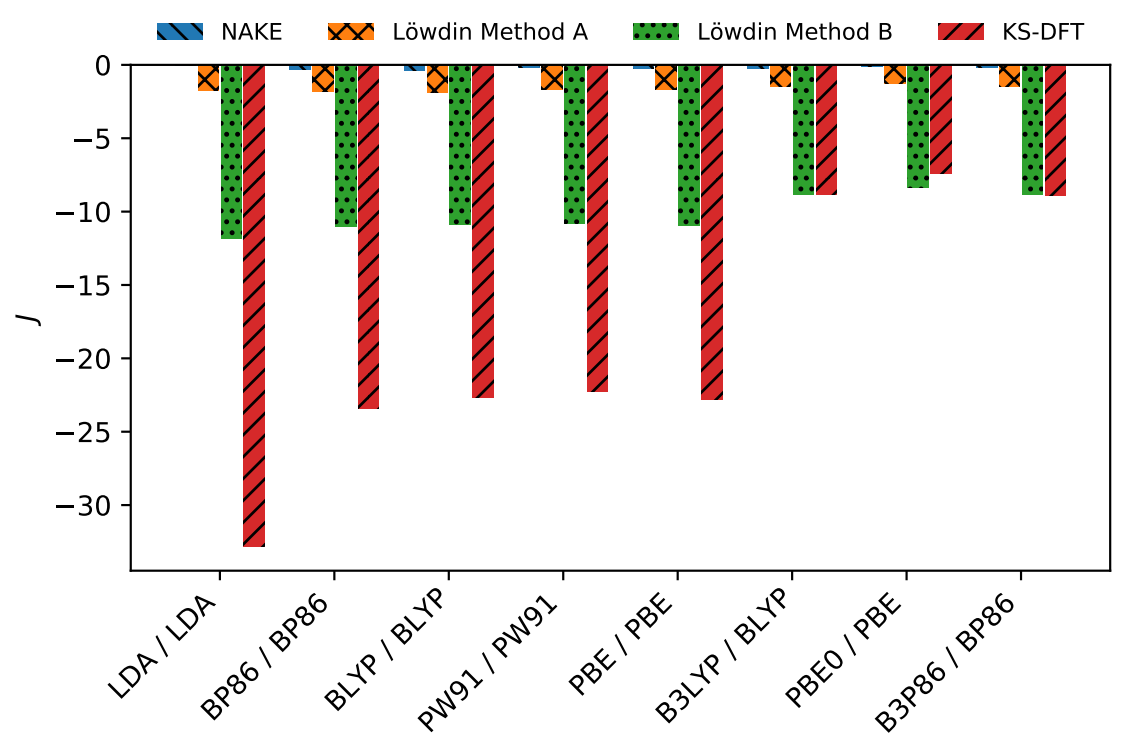

Figure S9: Magnetic exchange coupling constants evaluated for compound 3 with different schemes for the calculation of $T_{s}^{\text {nad }}$ and various XC / nad XC functionals. NAKE: $T_{s}^{\text {nad }}$ evaluated with a NAKE functional and Löwdin: $T_{s}^{\text {nad }}$ evaluated from Löwdin orthogonalized orbitals via method A or B. The corresponding KS-DFT values calculated with the listed XC functional are shown for reference. 


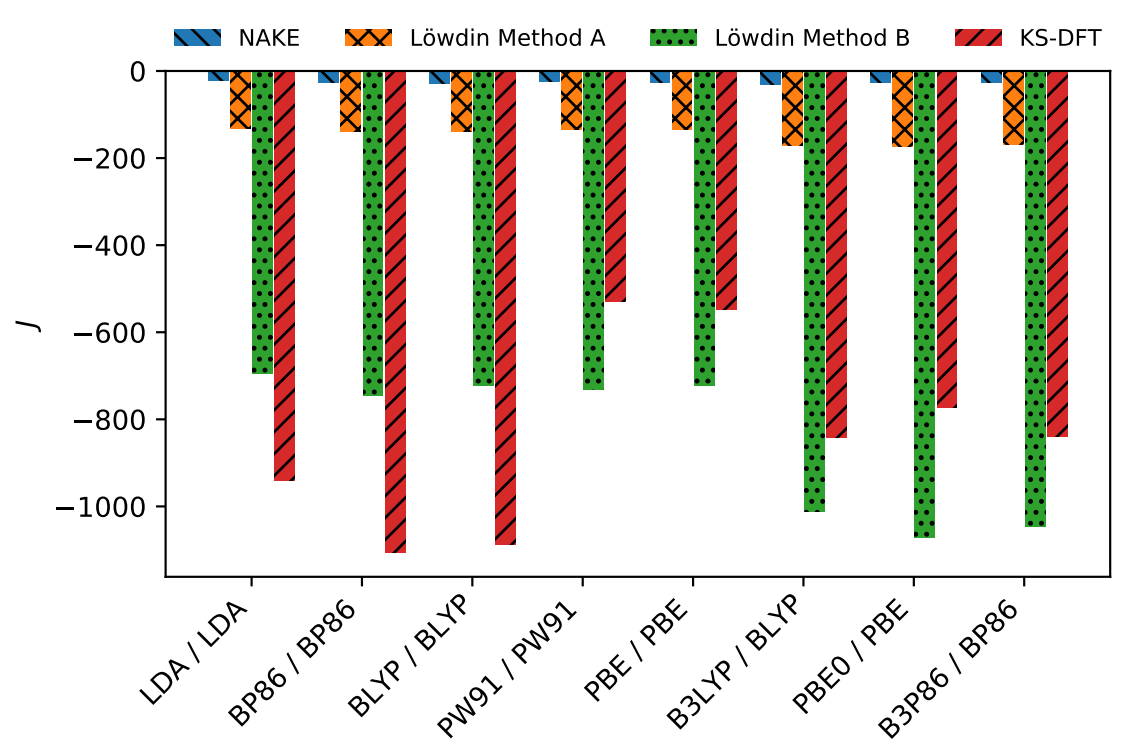

Figure S10: Magnetic exchange coupling constants evaluated for compound 4 with different schemes for the calculation of $T_{s}^{\text {nad }}$ and various XC / nad XC functionals. NAKE: $T_{s}^{\text {nad }}$ evaluated with a NAKE functional and Löwdin: $T_{s}^{\text {nad }}$ evaluated from Löwdin orthogonalized orbitals via method A or B. The corresponding KS-DFT values calculated with the listed XC functional are shown for reference.

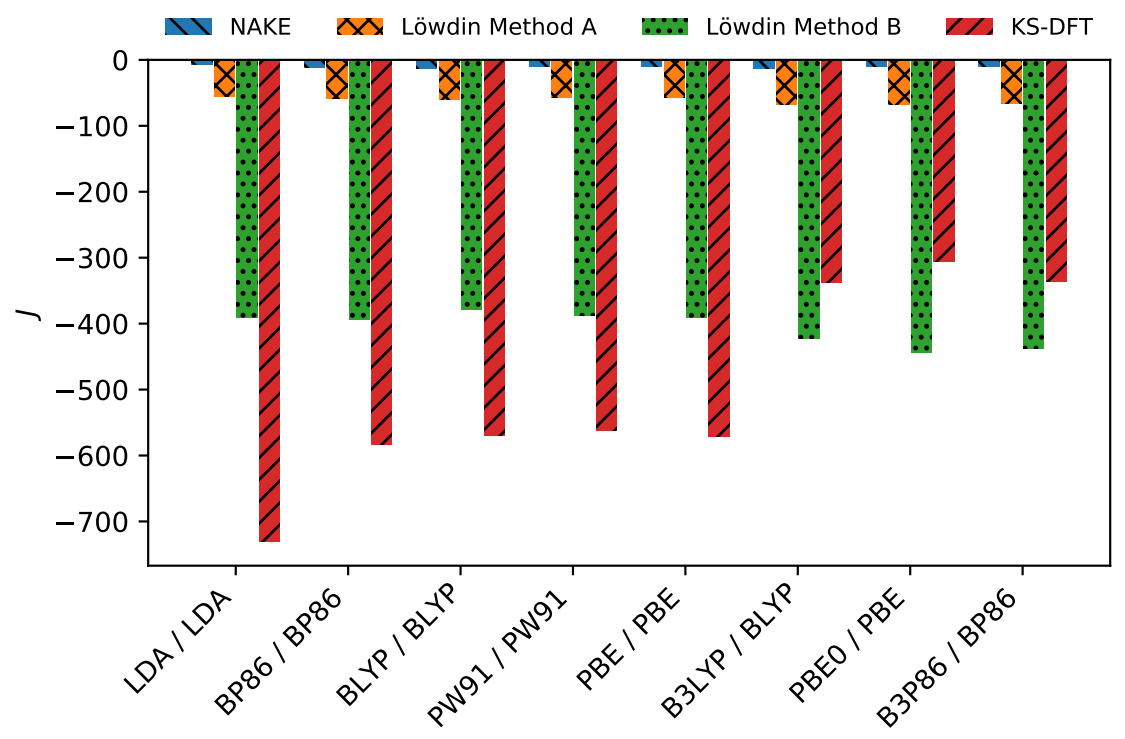

Figure S11: Magnetic exchange coupling constants evaluated for compound $\mathbf{5}$ with different schemes for the calculation of $T_{s}^{\text {nad }}$ and various XC / nad XC functionals. NAKE: $T_{s}^{\text {nad }}$ evaluated with a NAKE functional and Löwdin: $T_{s}^{\text {nad }}$ evaluated from Löwdin orthogonalized orbitals via method A or B. The corresponding KS-DFT values calculated with the listed XC functional are shown for reference. 


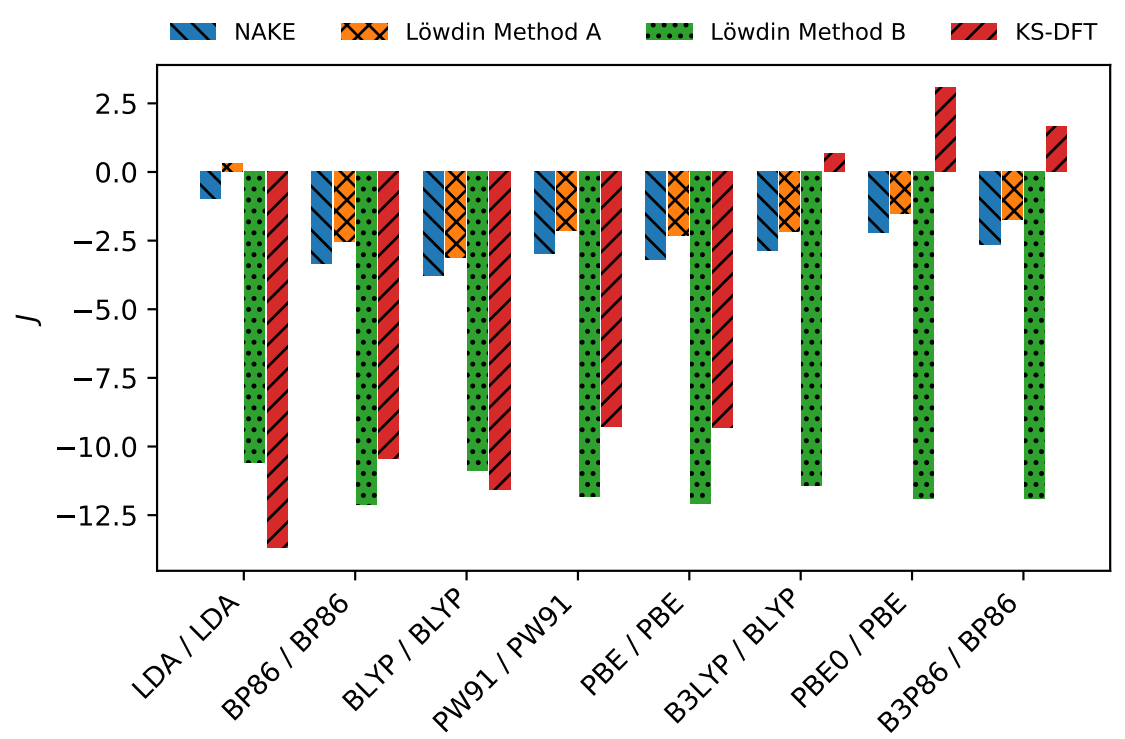

Figure S12: Magnetic exchange coupling constants evaluated for compound 6 with different schemes for the calculation of $T_{s}^{\text {nad }}$ and various XC / nad XC functionals. NAKE: $T_{s}^{\text {nad }}$ evaluated with a NAKE functional and Löwdin: $T_{s}^{\text {nad }}$ evaluated from Löwdin orthogonalized orbitals via method A or B. The corresponding KS-DFT values calculated with the listed XC functional are shown for reference.

\section{S5 Basis-Set Dependency}

To further investigate the basis-set dependency of the sDFT calculations the difference in the spin density introduced by a change of the basis-set is investigated. Figure S13 shows the difference in the spin density introduced by a change of the basis-set from def2-TZVP to def2-SVP. In case of the sDFT and KS-DFT calculations a change in the spin density is visible. The influence of the basis-set on the spin density is large compared to the spin density difference introduced by the orthogonalization procedure, see Fig. 3 in the main article (the isovalue used in Fig. 3 is a power of ten smaller). 
HS
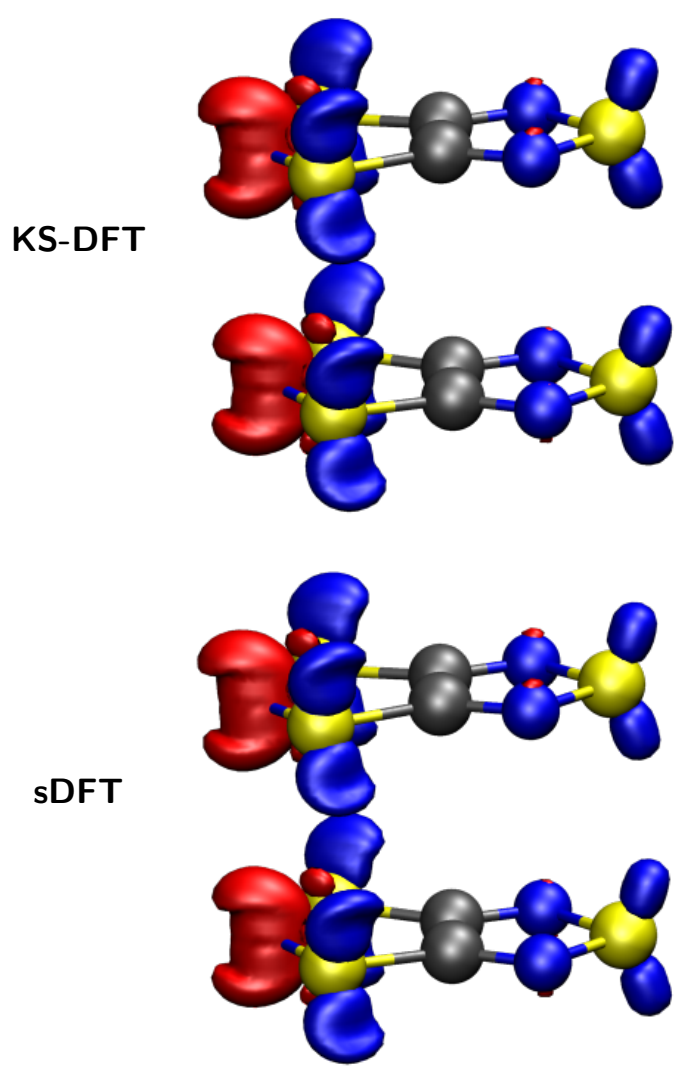

BS
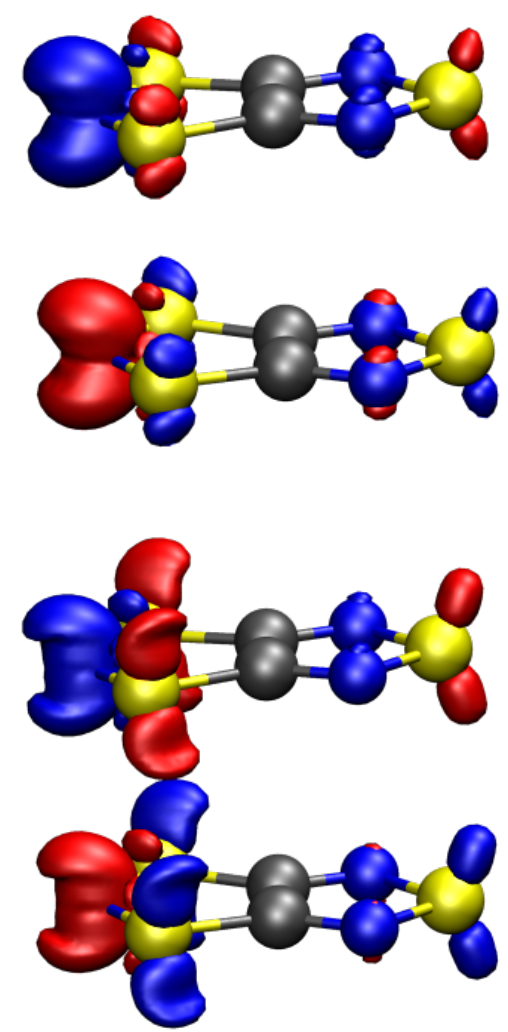

Figure S13: Spin density difference between the def2-TZVP and def2-SVP basis-set of the HS (left) and BS (right) state of dimer 1. The PBE0/PBE/PW91k functional combination was used for the sDFT calculations (bottom) while the PBE0 XC-functional was employed in the KS-DFT calculations (top). An isovalue of $0.001 E_{h}$ was applied.

The magnetic exchange coupling constants shown in Tab. 6 in the main article indicate an increase of the absolute value by about $50 \%$ when the def2-TZVP basis-set is used instead of the def2-SVP one. In Tab. S8 to S12 the BS-HS energy differences of the electrostatic, kinetic, XC and total energy contributions are listed for the def2-SVP and def2-TZVP basis-set. 
Table S7: Electrostatic, kinetic, XC and total energy differences between the BS and HS state of dimer 1 for the def2-SVP and def2-TZVP basis-set and the PBE0/PBE/PW91k functional combination. All energy differences are given in $E_{h}$.

\begin{tabular}{|c|c|c|c|c|c|c|}
\hline & \multirow{3}{*}{$\begin{array}{c}\text { KS-DFT } \\
\text { PBE0 }\end{array}$} & \multicolumn{5}{|c|}{ Subsystem DFT } \\
\hline & & \multirow[t]{2}{*}{ NAKE } & \multicolumn{2}{|c|}{ Pot. Rec. } & \multicolumn{2}{|c|}{ Löwdin } \\
\hline & & & Method A & Method B & Method A & Method B \\
\hline \multicolumn{7}{|c|}{ def2-SVP } \\
\hline$\Delta E_{\text {electrostatic }}^{\mathrm{BS}-\mathrm{HS}}$ & 0.081603 & 0.002947 & 0.007750 & 0.002947 & 0.012552 & 0.002947 \\
\hline$\Delta E_{\mathrm{kin}}^{\mathrm{BS}-\mathrm{HS}}$ & -0.110161 & -0.003692 & -0.013894 & -0.013894 & -0.016074 & -0.016074 \\
\hline$\Delta E_{\mathrm{XC}}^{\mathrm{BS}-\mathrm{HS}}$ & 0.020722 & 0.000463 & 0.000726 & 0.000463 & 0.001804 & 0.000465 \\
\hline$\Delta \mathrm{E}_{\text {total }}^{\mathrm{BS}-\mathrm{HS}}$ & -0.007836 & -0.000282 & -0.005418 & -0.010484 & -0.001719 & -0.012662 \\
\hline \multicolumn{7}{|c|}{ def2-TZVP } \\
\hline$\Delta E_{\text {electrostatic }}^{\mathrm{BS}-\mathrm{HS}}$ & 0.090488 & 0.004135 & -0.000301 & 0.004135 & 0.019507 & 0.004135 \\
\hline$\Delta E_{\mathrm{kin}}^{\mathrm{BS}-\mathrm{HS}}$ & -0.125424 & -0.005599 & -0.007072 & -0.007072 & -0.025186 & -0.025186 \\
\hline$\Delta E_{\mathrm{XC}}^{\mathrm{BS}-\mathrm{HS}}$ & 0.025627 & 0.001038 & 0.000790 & 0.001038 & 0.003247 & 0.001039 \\
\hline$\Delta \mathrm{E}_{\text {total }}^{\mathrm{BS}-\mathrm{HS}}$ & -0.009309 & -0.000427 & -0.006584 & -0.001899 & -0.002432 & -0.020011 \\
\hline
\end{tabular}

Table S8: Electrostatic, kinetic, XC and total energy differences between the BS and HS state of dimer 2 for the def2-SVP and def2-TZVP basis-set and the PBE0/PBE/PW91k functional combination. All energy differences are given in $E_{h}$.

\begin{tabular}{|c|c|c|c|}
\hline & \multicolumn{3}{|c|}{ Subsystem DFT } \\
\hline & NAKE & Method A & Method B \\
\hline \multicolumn{4}{|c|}{ def2-SVP } \\
\hline$\Delta E_{\text {electrostatic }}^{\mathrm{BS}-\mathrm{HS}}$ & 0.000529 & 0.001023 & 0.000529 \\
\hline$\Delta E_{\mathrm{kin}-\mathrm{HS}}^{\mathrm{BS}}$ & -0.000657 & -0.001247 & -0.001247 \\
\hline$\Delta E_{\mathrm{XC}}^{\mathrm{BS}-\mathrm{HS}}$ & 0.000089 & 0.000146 & 0.000089 \\
\hline$\Delta \mathrm{E}_{\text {total }}^{\mathrm{BS}-\mathrm{HS}}$ & -0.000040 & -0.000078 & -0.000629 \\
\hline \multicolumn{4}{|c|}{ def2-TZVP } \\
\hline$\Delta E_{\text {electrostatic }}^{\mathrm{BS}}$ & 0.001003 & 0.002453 & 0.001003 \\
\hline$\Delta E_{\mathrm{kin}-\mathrm{HS}}^{\mathrm{BS}}$ & -0.001371 & -0.003093 & -0.003093 \\
\hline$\Delta E_{\mathrm{XC}}^{\mathrm{BS}-\mathrm{HS}}$ & 0.000294 & 0.000472 & 0.000293 \\
\hline$\Delta \mathrm{E}_{\text {total }}^{\mathrm{BS}-\mathrm{HS}}$ & -0.000074 & -0.000169 & -0.001797 \\
\hline
\end{tabular}


Table S9: Electrostatic, kinetic, XC and total energy differences between the BS and HS state of dimer 3 for the def2-SVP and def2-TZVP basis-set and the PBE0/PBE/PW91k functional combination. All energy differences are given in $E_{h}$.

\begin{tabular}{lrrr}
\hline \hline & \multicolumn{3}{c}{ Subsystem DFT } \\
& \multicolumn{4}{c}{ Method A } & Method B \\
\hline \multicolumn{4}{c}{ def2-SVP } \\
\hline$\Delta E_{\text {electrostatic }}^{\mathrm{BS}-\mathrm{HS}}$ & 0.000009 & 0.000035 & 0.000009 \\
$\Delta E_{\text {kin }}^{\mathrm{BS}-\mathrm{HS}}$ & -0.000012 & -0.000049 & -0.000049 \\
$\Delta E_{\mathrm{XC}}^{\mathrm{BS}-\mathrm{HS}}$ & 0.000003 & 0.000008 & 0.000003 \\
\hline \hline$\Delta \mathrm{E}_{\text {total }}^{\mathrm{BS}-\mathrm{HS}}$ & -0.000001 & -0.000006 & -0.000038 \\
\hline \multicolumn{4}{c}{ def2-TZVP } \\
\hline$\Delta E_{\text {electrostatic }}^{\mathrm{BS}-\mathrm{HS}}$ & -0.000020 & -0.000015 & -0.000020 \\
$\Delta E_{\text {kin }}^{\mathrm{BS}-\mathrm{HS}}$ & 0.000022 & 0.000011 & 0.000011 \\
$\Delta E_{\text {XC }}^{\mathrm{BS}-\mathrm{HS}}$ & 0.000001 & 0.000003 & 0.000001 \\
\hline \hline$\Delta \mathrm{E}_{\text {total }}^{\mathrm{BS}-\mathrm{HS}}$ & 0.000002 & -0.000001 & -0.000009 \\
\hline \hline
\end{tabular}

Table S10: Electrostatic, kinetic, XC and total energy differences between the BS and HS state of dimer 4 for the def2-SVP and def2-TZVP basis-set and the PBE0/PBE/PW91k functional combination. All energy differences are given in $E_{h}$.

\begin{tabular}{lrrr}
\hline \hline & \multicolumn{3}{c}{ Subsystem DFT } \\
& \multicolumn{4}{c}{ Method A } & Method B \\
\hline \multicolumn{4}{c}{ def2-SVP } \\
\hline$\Delta E_{\text {electrostatic }}^{\text {BS-HS }}$ & 0.000883 & 0.004310 & 0.000883 \\
$\Delta E_{\text {kin }}^{\text {SS }}$ & -0.001243 & -0.006043 & -0.006043 \\
$\Delta E_{\text {XC }}^{\mathrm{BS}-H S}$ & 0.000238 & 0.000933 & 0.000241 \\
\hline \hline$\Delta \mathbf{E}_{\text {total }}^{\text {BS-HS }}$ & -0.000123 & -0.000799 & -0.004919 \\
\hline \multicolumn{4}{c}{ def2-TZVP } \\
\hline$\Delta E_{\text {electrostatic }}^{\text {BS-HS }}$ & 0.000826 & 0.005967 & 0.000826 \\
$\Delta E_{\text {kin }}^{\text {BS-HS }}$ & -0.001410 & -0.008459 & -0.008459 \\
$\Delta E_{\text {XC }}^{\text {BS-HS }}$ & 0.000440 & 0.001431 & 0.000442 \\
\hline \hline$\Delta \mathbf{E}_{\text {total }}^{\text {BS-HS }}$ & -0.000144 & -0.001060 & -0.007190 \\
\hline \hline
\end{tabular}


Table S11: Electrostatic, kinetic, XC and total energy differences between the BS and HS state of dimer $\mathbf{5}$ for the def2-SVP and def2-TZVP basis-set and the PBE0/PBE/PW91k functional combination. All energy differences are given in $E_{h}$.

\begin{tabular}{lrrr}
\hline \hline & \multicolumn{3}{c}{ Subsystem DFT } \\
& \multicolumn{4}{c}{ Method A } & Method B \\
\hline \multicolumn{4}{c}{ def2-SVP } \\
\hline$\Delta E_{\text {electrostatic }}^{\mathrm{BS}-\mathrm{HS}}$ & 0.000362 & 0.001768 & 0.000362 \\
$\Delta E_{\text {kin } \mathrm{BS}}^{\mathrm{BS}}$ & -0.000511 & -0.002496 & -0.002496 \\
$\Delta E_{\mathrm{XC}}^{\mathrm{BS}-\mathrm{HS}}$ & 0.000102 & 0.000419 & 0.000102 \\
\hline \hline$\Delta \mathbf{E}_{\text {total }}^{\mathrm{BS}-\mathrm{HS}}$ & -0.000046 & -0.000309 & -0.002031 \\
\hline \multicolumn{4}{c}{ def2-TZVP } \\
\hline$\Delta E_{\text {electrostatic }}^{\mathrm{BS}-\mathrm{TS}}$ & 0.000358 & 0.002706 & 0.000358 \\
$\Delta E_{\text {kin }}^{\mathrm{BS}-\mathrm{SS}}$ & -0.000617 & -0.003818 & -0.003818 \\
$\Delta E_{\text {XC }}^{\mathrm{BS}-\mathrm{HS}}$ & 0.000203 & 0.000689 & 0.000196 \\
\hline \hline$\Delta \mathbf{E}_{\text {total }}^{\mathrm{BS}-\mathrm{HS}}$ & -0.000057 & -0.000422 & -0.003263 \\
\hline \hline
\end{tabular}

Table S12: Electrostatic, kinetic, XC and total energy differences between the BS and HS state of dimer 6 for the def2-SVP and def2-TZVP basis-set and the PBE0/PBE/PW91k functional combination. All energy differences are given in $E_{h}$.

\begin{tabular}{lrrr}
\hline \hline & \multicolumn{3}{c}{ Subsystem DFT } \\
& \multicolumn{4}{c}{ Method A } & Method B \\
\hline \multicolumn{4}{c}{ def2-SVP } \\
\hline$\Delta E_{\text {electrostatic }}^{\text {BS-HS }}$ & 0.000081 & 0.000117 & 0.000081 \\
$\Delta E_{\text {kin }}^{\text {BS }}$ & -0.000114 & -0.000158 & -0.000158 \\
$\Delta E_{\text {XC }}^{\text {BS }}$ & 0.000023 & 0.000034 & 0.000023 \\
\hline \hline$\Delta \mathbf{E}_{\text {total }}^{\text {BS-HS }}$ & -0.000010 & -0.000007 & -0.000054 \\
\hline & def2-TZVP & \\
\hline$\Delta E_{\text {electrostatic }}^{\text {BS-HS }}$ & 0.000016 & -0.000019 & 0.000016 \\
$\Delta E_{\text {kin }}^{\text {BS-HS }}$ & -0.000034 & 0.000013 & 0.000013 \\
$\Delta E_{\text {XC }}^{\text {BS-HS }}$ & 0.000020 & 0.000011 & 0.000018 \\
\hline \hline$\Delta \mathbf{E}_{\text {total }}^{\text {BS-HS }}$ & 0.000002 & 0.000005 & 0.000047 \\
\hline \hline
\end{tabular}




\section{S6 Geometric Dependency - Orbitals}

The heat maps in Fig. 6 in the main article show a sign change of the coupling constant at $\Delta h=1.8,2.4 \AA$ and $3.0 \AA$. The SOMO orbitals of the HS and BS state with $\Delta v=0.0 \AA$ and $\Delta h=0.0,1.2,1.8,2.4 \AA$ and $3.0 \AA$ are shown in Fig. $\mathrm{S} 14$.

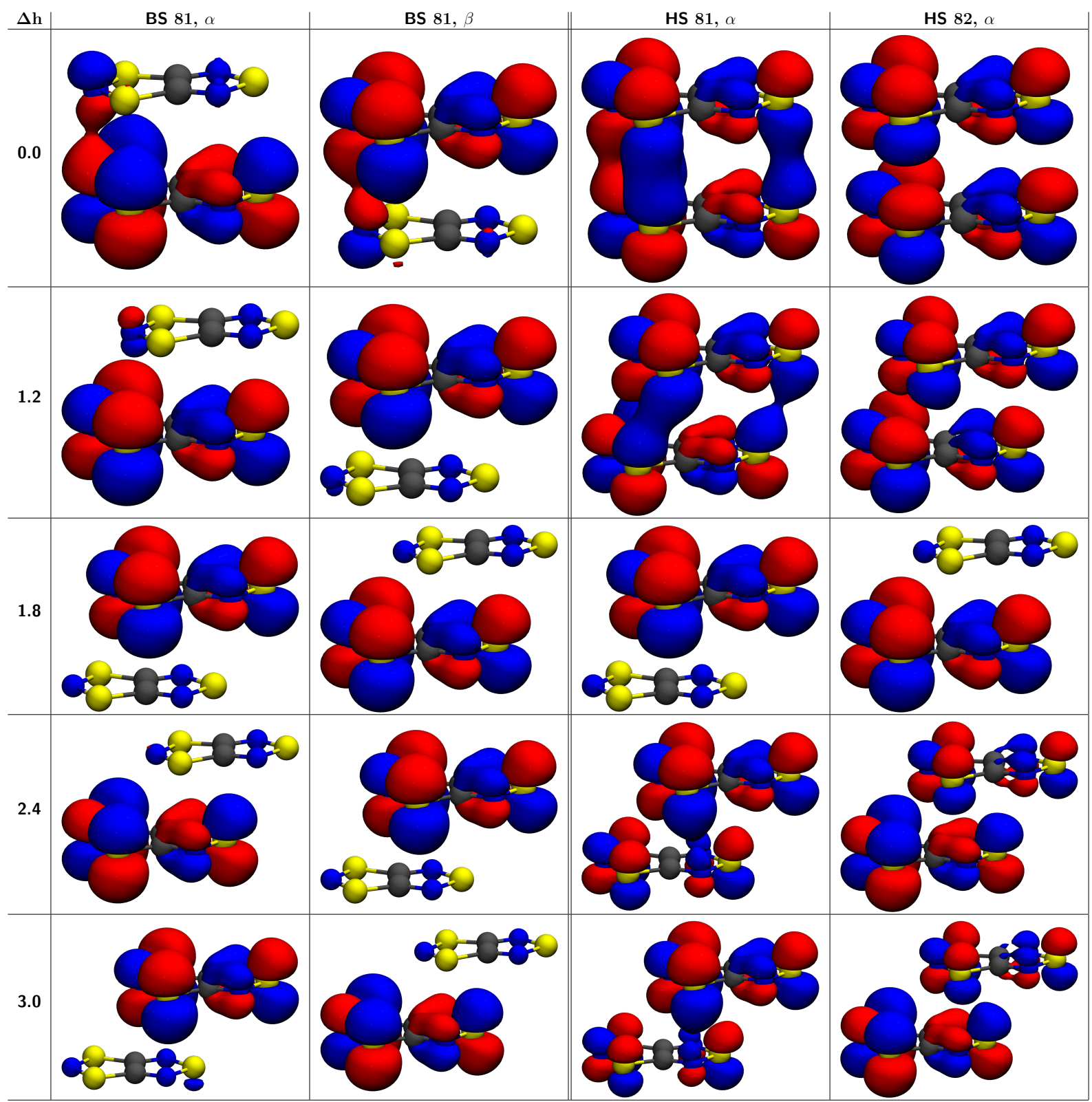

Figure S14: SOMO orbitals of dimer 1 for different $\Delta h$ and $\Delta v=0.0 \AA$ calculated with KS-DFT employing the PBE0 XC-functional and def2-SVP basis set for the HS and BS state. An isovalue of 0.025 a.u. was applied. 


\section{S7 Interaction Energy}

The intermolecular interaction energy was calculated via KS-DFT using the PBE0 XC functional and def2-TZVP basis-set without and with counterpoise correction, see Tab. S13 and Tab. S14. Dimer 1 has the strongest interaction in the HS state.

Table S13: Intermolecular interaction energy of the radical pairs, calculated with PBE0 KS-DFT as $E_{\text {Dimer }}-E_{\text {Monomer 1 }}-E_{\text {Monomer } 2}$ employing the def2-TZVP basis-set. The monomer structures are fixed from the dimer structure.

\begin{tabular}{rrr}
\hline \hline & $\mathrm{HS} / E_{h}$ & $\mathrm{BS} / E_{h}$ \\
\hline $\mathbf{1}$ & 0.0156 & 0.0063 \\
$\mathbf{2}$ & 0.0015 & 0.0009 \\
$\mathbf{3}$ & -0.0036 & -0.0036 \\
$\mathbf{4}$ & -0.0016 & -0.0057 \\
$\mathbf{5}$ & -0.0021 & -0.0036 \\
$\mathbf{6}$ & -0.0155 & -0.0155 \\
\hline \hline
\end{tabular}

Table S14: Intermolecular interaction energy of the radical pairs, calculated with PBE0 KS-DFT

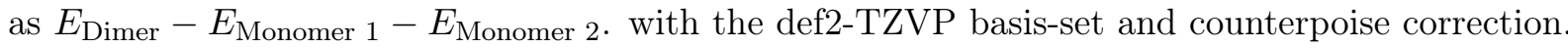
The monomer structures are fixed from the dimer structure.

\begin{tabular}{rrr}
\hline \hline & $\mathrm{HS} / E_{h}$ & $\mathrm{BS} / E_{h}$ \\
\hline $\mathbf{1}$ & 0.0167 & 0.0074 \\
$\mathbf{2}$ & 0.0024 & 0.0018 \\
$\mathbf{3}$ & -0.0030 & -0.0030 \\
$\mathbf{4}$ & 0.0010 & -0.0031 \\
$\mathbf{5}$ & -0.0004 & -0.0019 \\
$\mathbf{6}$ & -0.0148 & -0.0147 \\
\hline \hline
\end{tabular}

The values in the heat maps shown in Fig. 6 in the main article posses the same values within the reported precision at $1.8 \AA$, but there are actually small differences as shown in Tab. S15 
Table S15: Geometric dependency of the magnetic exchange coupling constants with $\Delta h=$ $1.8 \AA$, see also Fig. 6 in the main article.

\begin{tabular}{rrrrr}
\hline \hline$\Delta v$ & $\Delta h$ & KS-DFT & Isolated & FAT \\
\hline $0.2 \AA$ & $1.8 \AA$ & $7.49 \mathrm{~cm}^{-1}$ & $7.42 \mathrm{~cm}^{-1}$ & $6.01 \mathrm{~cm}^{-1}$ \\
$0.1 \AA$ & $1.8 \AA$ & $9.77 \mathrm{~cm}^{-1}$ & $10.15 \mathrm{~cm}^{-1}$ & $8.12 \mathrm{~cm}^{-1}$ \\
$0.0 \AA$ & $1.8 \AA$ & $12.51 \mathrm{~cm}^{-1}$ & $13.19 \mathrm{~cm}^{-1}$ & $10.54 \mathrm{~cm}^{-1}$ \\
$-0.1 \AA$ & $1.8 \AA$ & $15.72 \mathrm{~cm}^{-1}$ & $16.31 \mathrm{~cm}^{-1}$ & $13.15 \mathrm{~cm}^{-1}$ \\
$-0.2 \AA$ & $1.8 \AA$ & $19.16 \mathrm{~cm}^{-1}$ & $19.08 \mathrm{~cm}^{-1}$ & $15.36 \mathrm{~cm}^{-1}$ \\
\hline \hline
\end{tabular}

S8 $\left\langle\mathbf{S}^{2}\right\rangle$ Values

The $\left\langle S^{2}\right\rangle$ values of the HS and BS state for the KS-DFT calculations of compound $\mathbf{6}$ are shown in Fig. S15.

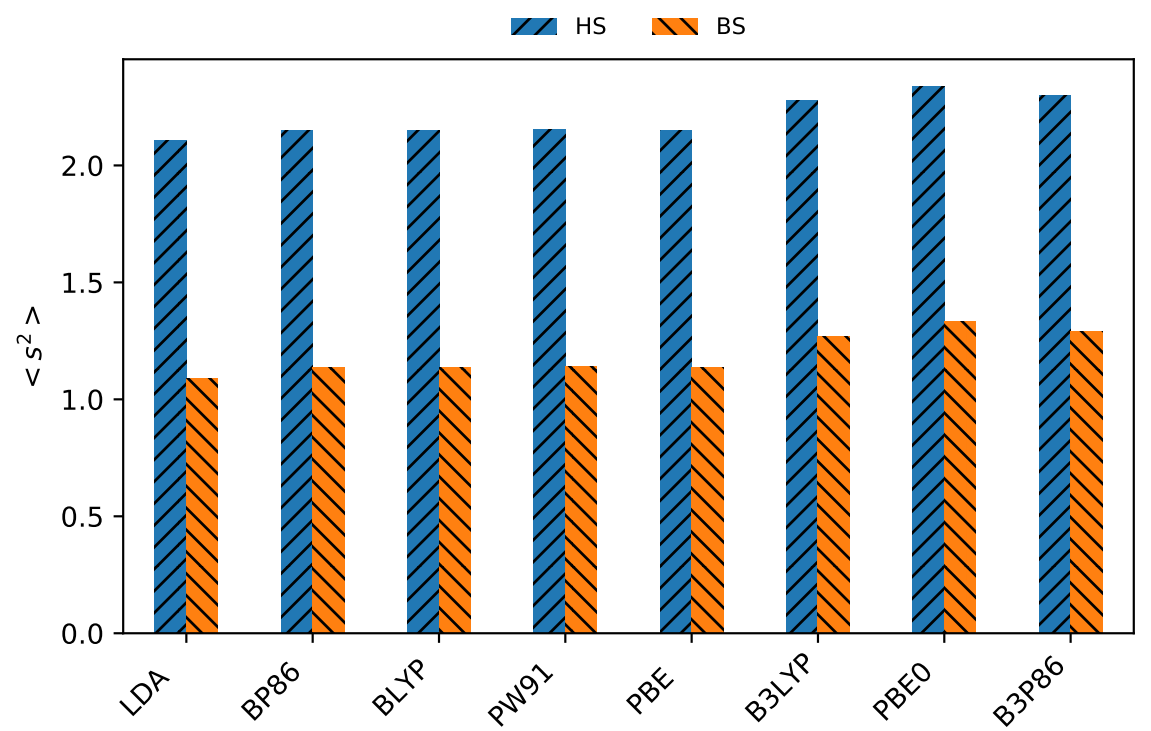

Figure S15: KS-DFT calculated $\langle S\rangle$ values of compound 6 for the HS and BS state. 


\section{References}

[1] Löwdin, P.-O. On the Non-Orthogonality Problem Connected with the Use of Atomic Wave Functions in the Theory of Molecules and Crystals. The Journal of Chemical Physics 1950, 18, 365-375.

[2] Pipek, J. Controlled Orthogonalization of Localized Orbitals. Int. J. Quantum Chem. 1985, 27, 527-546.

[3] Broer, R. On the Use of Corresponding Orbitals for the Construction of Mutually Orthogonal Orbital Sets. Int. J. Quantum Chem. 1993, 45, 587-590.

[4] Mo, Y.; Peyerimhoff, S. D. Theoretical Analysis of Electronic Delocalization. J. Chem. Phys. 1998, 109, 1687-1697. 